\title{
Caractérisation de 118 variétés de cotonnier (Gossypium hirsutum) en condition de déficit hydrique au Togo
}

\author{
Kokou Z. KOFFI ${ }^{1 *}$, Nambou GNOFAM ${ }^{1}$, Komlan P. AKANTETOU ${ }^{1}$, \\ Mawuli AZIADEKEY ${ }^{2}$ et Koffi TOZO ${ }^{3}$ \\ ${ }^{1}$ Centre de Recherche Agronomique de la Savane Humide de Institut Togolais de Recherche Agronomique \\ (CRA-SH/ITRA), 220 BP 01 Anié, Togo. \\ ${ }^{2}$ Laboratoire de l'interface Sciences du Sol-Climat-Production Végétale, Université de Lomé, 01 BP 1515, \\ Lomé 01, Togo. \\ ${ }^{3}$ Laboratoire de Physiologie et de Biotechnologie des Plants, Université de Lomé, 01 BP 1515, Lomé 01, Togo. \\ *Auteur correspondant ; E-mail: kofzovodu@yahoo.fr; Tel. (+228) 92326101
}

\begin{tabular}{lll}
\hline Received: 07-05-2021 & Accepted: 15-10-2021 & Published: 30-10-2021 \\
\hline
\end{tabular}

\section{RESUME}

Le déficit hydrique est le principal facteur abiotique qui limite la productivité des cultures dans les conditions de l'agriculture pluviale. L'objectif de cette étude a été d'identifier les variétés de cotonnier (Gossypium. hirsutum) tolérantes au déficit hydrique au Togo. Pour cela, 117 accessions et une variété témoin de G. hirsutum ont été caractérisées sous les régimes hydriques optimal et déficitaire. Le dispositif expérimental a été un Alpha lattice avec 120 objets en trois répétitions. Onze caractéristiques agromorphologiques ont été mesurées. Les indices de sensibilité (DSI) et de tolérance (STI) au déficit ont été calculés. Les résultats ont révélé une grande variabilité au déficit hydrique au sein des accessions testées. Le déficit hydrique a entrainé une réduction du rendement en coton graine de $85 \%$. Par ailleurs, nous avons noté qu'en condition de déficit, le rendement a été corrélé négativement avec le DSI et positivement avec les indices STI et GMP. Quatre groupes de variétés ont été distingués. Il s'agit des variétés tolérantes à haut ou faible potentiel de production et des variétés sensibles à haut ou faible potentiel de production. Les variétés tolérantes à haut potentiel de production constituent une source importante de géniteurs, pour l'amélioration de la résistance au déficit hydrique des variétés vulgarisées au Togo.

(C) 2021 International Formulae Group. All rights reserved.

Mots clés : Sécheresse, tolérance, cotonnier, variétés, Togo.

\section{Characterization of 118 cotton varieties (Gossypium hirsutum) under water deficit conditions in Togo}

\begin{abstract}
Drought is the major abiotic factor limiting crop productivity in rainfed agriculture conditions. This study aimed to identify cotton varieties (Gossypium hirsutum) tolerant to water deficit in Togo. For this purpose, 117 accessions of $G$. hirsutum and one check variety were characterized under optimal and deficit water conditions. The check variety was repeated three times to have 120 objects. The experiment was arranged in Alpha lattice
\end{abstract}


design with 120 objects and three replications. Eleven agromorphological traits were measured. The water deficit susceptibility index (DSI) and tolerance index (STI) were calculated. The results revealed a high variability within the tested accessions to water deficit. The water deficit resulted in a $85 \%$ reduction in seed cotton yield due to a decrease in the number of capsules per plant and average capsular weight. The results also showed that, under deficit conditions, yield was negatively correlated with DSI and positively correlated with STI. Four groups of varieties were distinguished. These were tolerant varieties with high or low production potential; susceptible varieties with high or low production potential. The tolerant varieties with high production potential constitute an important source of genes for the improvement of the resistance to water deficit of the cotton cultivars of Togo.

(C) 2021 International Formulae Group. All rights reserved.

Keywords: Drought, tolerance, cotton, varieties, Togo.

\section{INTRODUCTION}

Le coton est une culture stratégique pour les pays de l'Afrique de l'Ouest (Bakayoko, 2013). L'exportation de la fibre constitue une source importante de devise pour ces pays et la graine est utilisée en alimentation humaine (huile) ou des animaux (tourteaux). $\mathrm{Au}$ Togo, le coton constitue le premier produit agricole d'exportation qui procure d'importantes devises pour le pays et des revenus sûrs pour les producteurs (MAEP, 2013). Cependant, cette culture est soumise à de nombreuses contraintes biotiques (maladies et ravageurs) et abiotiques (dégradation du sol, salinité du sol, déficit hydrique etc.). Parmi les contraintes abiotiques, l'une des principales est la sécheresse qui survient au cours du cycle de la culture (Sultan et al., 2008 ; Lacape et al., 2015). La sécheresse entraine une baisse de la production en quantité et en qualité des cultures (Karademir et al., 2011 ; Lacape et al., 2015 ; Akata, 2018).

$\mathrm{Au}$ Togo, les études de Djaman et al. (2017) ont révélées une tendance à la baisse de la quantité de pluies annuelles dans les différentes zones agroécologiques du pays. Contrairement, Koudahé et al. (2018) et Gadedjisso-Tossou et al. (2020) ont trouvé une tendance à l'augmentation des pluies au sud et au nord du Togo respectivement. Même si la tendance est à l'augmentation, la mauvaise répartition constitue un sérieux problème. Adewi et al. (2010) ont révélé des perturbations pluviométriques au Togo, marquées par le début tardif et la fin précoce des pluies avec de longues poches de sécheresse. Pour ce qui est des températures, Koudahe et al. (2018) ont trouvé une tendance à l'augmentation des températures minimales (Tmin) et maximales (Tmax) au Sud du Togo. Mais, au Nord du Togo, Gadedjisso-Tossou et al. (2020) ont trouvé une tendance à l'augmentation de la Tmax à Kara, Niamtougou et Mango et, une tendance à la baisse de la Tmax à Dapaong.

Pour minimiser les impacts négatifs de la sécheresse sur la production agricole, plusieurs stratégies ont été développées. Il s'agit des pratiques culturales favorisant l'augmentation de la capacité de rétention d'eau du sol et/ou du développement du système racinaire des plants (semis sous couvert végétal, apport du compost, du fumier et/ou de l'engrais minéral) (Sissoko et al., 2013 ; Some et al., 2014 ; Bokobana et al., 2019 ; Sissoko et al., 2020). A ces pratiques nous pouvons ajouter les dates de semis, la densité de semis, l'application des régulateurs de croissance et l'utilisation de variétés tolérantes aux stress hydriques (Ouédraogo et al., 2010 ; Khan et al., 2018). Parmi toutes ses stratégies, seule la création variétale n'entraine pas des coûts supplémentaires de production aux producteurs qui, dans les pays en voie de développement, arrivent difficilement à supporter les coûts déjà existants.

De nombreux travaux de recherche ont montré qu'il existe chez le cotonnier une variabilité de réponses au déficit hydrique. Des variétés sont capables (i) d'esquiver le stress hydrique en raccourcissant ou en allongeant leur cycle de développement; (ii) d'éviter le stress en réduisant la perte d'eau par régulation des ouvertures des stomates et/ou en augmentant la disponibilité en eau par 
allongement du système racinaire; (iii) de tolérer le déficit par des phénomènes d'osmorégulation (Al-Khayri et al., 2015; Akata, 2018).

$\mathrm{Au}$ Togo, les travaux de création variétale menés jusqu'à présent sur le cotonnier ont porté essentiellement sur l'amélioration des caractéristiques agronomiques, technologiques et de la résistance au facteurs biotiques (maladies et ravageurs) (Schwartz, 1985; Koffi et al., 2017 ; Koffi et al., 2019). Peu de travaux ont porté sur les facteurs abiotiques. Gnofam et al. (2014) ont montré que la variété STAM 129A actuellement vulgarisée au Togo est sensible au déficit hydrique. Améliorer sa résistance au déficit constitue pour les sélectionneurs du pays un défi à relever pour sécuriser les revenus des producteurs. C'est dans cette optique que cette étude a été menée. L'objectif de ce travail est d'identifier parmi les variétés de cotonnier ( $G$. hirsutum) disponibles au Togo, celles qui sont tolérantes au déficit hydrique.

\section{MATERIEL ET METHODES Site de l'étude}

L'étude a été conduite sur la station de Kolokopé (N 747'56’, E 1¹7’38’') du Centre de Recherche Agronomique de la Savane Humide (CRA-SH).

Le site de notre étude (bande E2) est caractérisé par un sol de type sablo-limoneux avec un taux d'argile plus élevé au-delà des 20 $\mathrm{cm}$ de profondeur. Les caractéristiques moyennes physiques et hydriques du sol de la parcelle de l'essai dans 1'horizon 0-20 et 20$100 \mathrm{~cm}$ sont consignées dans le Tableau 1 suivant.

La réserve utile de ce sol a été en moyenne sur l'horizon $0-100 \mathrm{~cm}$ de $0,345 \mathrm{~mm}$ d'eau par cm d'épaisseur du sol soit $34,5 \mathrm{~mm}$ d'eau par mètre.

En condition optimale, l'essai a été conduit de juillet à décembre 2019. L'apport d'eau a été en majorité pluvial. Mais l'essai en condition de déficit a été mené pendant la grande saison sèche (04 novembre 2019 au 03 avril 2020) où l'apport d'eau a été effectué par arrosage.
Les données de température ont été obtenues grâce à la station de météorologie située à $150 \mathrm{~m}$ de notre parcelle d'étude.

En condition hydrique optimale, l'évolution des températures montre une légère augmentation de la température maximale et une stabilisation de la température minimale (Figure 1a). La température moyenne journalière a varié de $24,55^{\circ} \mathrm{C}$ à $31,05^{\circ} \mathrm{C}$. En condition de déficit hydrique, la variation de la température minimale a été beaucoup plus importante que celle de la température maximale (Figure 1b). Nous avons observé une baisse de la température minimale du semis au $60^{\text {ème }}$ jour après semis (j.a.s.), ensuite, une augmentation du $60^{\text {ème }}$ au $70^{\text {ème }}$ j.a.s., suivi d'une baisse jusqu'au $90^{\text {ème }}$ j.a.s. et en fin, une augmentation jusqu'au $120^{\text {ème }}$ j.a.s. suivi d'une faible fluctuation jusqu'à la récolte. $\mathrm{La}$ température moyenne a varié de $23,3{ }^{\circ} \mathrm{C}$ à 30,1 ${ }^{\circ} \mathrm{C}$ du semis à la floraison (100 j.a.s.) et de 26,2 ${ }^{\circ} \mathrm{C}$ à $33,3{ }^{\circ} \mathrm{C}$ de la floraison à l'ouverture complète des capsules.

Les températures enregistrées au cours des deux essais se situent dans les normes requises pour un développement optimal du cotonnier (Parry, 1982).

\section{Matériel végétal}

Le matériel végétal a été constitué de 117 variétés de cotonnier plus un témoin tous de G. hirsutum. Ces variétés sont issues d'Amérique du nord (USA), d'Amérique du sud (Brésil, Costa-Rica, Argentine), d'Asie (Ouzbékistan, Thaïlande), Europe (Turquie) et d'Afrique (Bénin, Burkina-Faso, Cameroun, Côte d'Ivoire, Mali, Tchad, Togo, Zambie et Zimbabwe).

\section{Dispositif expérimental}

Deux essais ont été implantés suivant le dispositif expérimental alpha lattice (Dagnelie, 2012) en trois répétitions. Afin d'équilibrer le dispositif, le témoin a été répété trois fois afin d'avoir 120 objets par répétition. Le nombre de blocs par répétition a été 12 et le nombre d'objets par bloc a été 10. Chaque parcelle élémentaire a été constituée d'une ligne de $4 \mathrm{~m}$ de long. Chaque parcelle a été semée aux 
écartements de $75 \mathrm{~cm}$ entre les lignes et de 30 $\mathrm{cm}$ entre les poquets sur la ligne soit au total 13 poquets par parcelle élémentaire. Les observations ont été réalisées sur chaque ligne.

\section{Régime hydrique}

Le premier essai a été conduit en condition hydrique optimale (OI) de juillet à novembre 2019. Les variétés ont été cultivées en condition pluviale, mais une irrigation d'appoint (14 $\mathrm{mm}$ d'eau) a été effectuée pendant la poche de sécheresse de la deuxième décade du mois d'août pour créer les conditions hydriques optimales de développement des cotonniers.

Le deuxième essai a été conduit en condition de déficit hydrique (DI) pendant l'intercampagne de novembre 2019 à avril 2020. Du semis à la floraison 243,94 mm d'eau ont été apporté aux plants. Dès l'apparition de la première fleur, l'apport d'eau a été interrompu pendant 30 jours pour simuler une poche de sécheresse. Ensuite, l'arrosage a été repris jusqu'à l'ouverture des capsules. La quantité d'eau apportée en fonction des différentes phases du cotonnier est mentionnée dans le Tableau 2 suivant.

\section{Conduite de l'essai}

Les parcelles des essais 1 et 2 ont été labourées respectivement le 25 juin et le 3 octobre 2019. Les semis des deux essais ont été effectués respectivement le 11 juillet 2019 et le 4 novembre 2019. Les apports des fumures minérales NPKSB à $200 \mathrm{~kg} /$ ha et de l'urée à 50 $\mathrm{kg} /$ ha ont été effectués respectivement le 20 ème et le $40^{\text {ème }}$ j.a.s.

Afin de limiter au maximum l'effet des ravageurs, vingt-quatre et vingt-six traitements insecticides ont été effectués dans l'essail et l'essai 2 respectivement. Le premier traitement a été effectué au $30^{\mathrm{e}} \mathrm{j}$.a.s après le semis pour le premier essai et au $20^{\mathrm{e}}$ j.a.s pour le second essai pour raison de l'attaque précoce des plants par les mouches blanches Bemisia tabaci.

Trois différents produits insecticides ont été utilisés dans le premier essai. Ces produits ont été (i) la combinaison de cyperméthrine $(72 \mathrm{~g} / \mathrm{l})$ et abamectine $20 \mathrm{~g} / \mathrm{l})$ à une dose de $500 \mathrm{ml} /$ ha pour lutter contre les chenilles carpophages et phyllophages et les piqueurs-suceurs ; (ii) l'indoxacarbe (150 g/l) à une dose de $170 \mathrm{ml} /$ ha pour lutter contre les chenilles carpophages exocarpiques et (iii) la combinaison de deltaméthrine $(24 \mathrm{~g} / \mathrm{l})$ et Acétamipride $(16 \mathrm{~g} / \mathrm{l})$ à la dose de $500 \mathrm{ml} / \mathrm{ha}$ pour lutter contre les pucerons, les mouches banches et les chenilles carpophages.

Les produits utilisés dans le second essai ont été la combinaison deltaméthrine (24 $\mathrm{g} / \mathrm{l})$ et pyriproxyfène $(60 \mathrm{~g} / \mathrm{l})$ pour mieux contrôler les mouches blanches et les trois combinaisons de produits utilisées dans le premier essai.

Deux sarclages $\left(15^{\text {ème }}\right.$ et $38^{\text {ème }}$ j.a.s $)$ et un buttage ( $72^{\text {ème }}$ j.a.s) ont été effectués dans le premier essai tandis que dans le second essai, 3 sarclages $\left(15^{\text {ème }}, 35^{\text {ème }}\right.$ et $\left.75^{\text {ème }}\right)$ ont été effectués.

\section{Paramètres observés ou calculés}

Les observations ont porté sur les caractéristiques morphologiques, phénologiques et agronomiques des variétés.

Les caractéristiques morphologiques ont été mesurées sur 5 plants choisis au hasard par parcelle élémentaire. Ces caractéristiques ont été la hauteur des plants à la floraison (HF) et à la récolte $(\mathrm{HR})$, le nombre de branches végétatives $(\mathrm{BV})$ et fructifères $(\mathrm{BF})$, le nombre de capsules portées par les branches végétatives (CBV), fructifères $(\mathrm{CBF})$ et le plant en entier (CT).

Les caractéristiques phénologiques ont été la date d'apparition de la première fleur (DFL1) et la précocité de la récolte.

La DFL1 a été évaluée par le comptage journalier des fleurs du jour. Est considéré comme la date d'apparition de la première fleur, le jour où le nombre de fleur compté a été égale au nombre de plant sur la ligne,

La précocité de la récolte a été calculée en faisant le rapport de la première récolte $(\mathrm{R} 1)$ effectuée à 125 j.a.s sur la récolte totale (RT).

Les caractéristiques agronomiques ont été le rendement en coton graine (RDT) calculé en extrapolant la production parcellaire à l'hectare et le poids moyen capsulaire (PMC). 
Le PMC a été déterminé par le rapport du poids de coton graine de capsules récoltées en première position sur les branches fructifères 3 , 4 ou 5 sur le nombre de capsules.

A partir des caractères mesurés et la production des deux essais, cinq indices suivants ont été calculés (Akata, 2018) :

- Indice de l'intensité du stress (SI), qui a été déterminé suivant la formule : $S I=1-\left[\frac{Y s t r}{Y Y_{\text {etm }}}\right]$, Ystr représente le poids de coton graine moyen en condition de déficit hydrique et Yetm, celui du contrôle bien irrigué. Cet indice est compris entre 0 et 1 ; plus il est élevé, plus sévère est le stress hydrique vécu par les plantes.

- Indice de sensibilité au stress (DSI), qui a été déterminé suivant la formule : $D S I=\frac{1-\left[\frac{y i s t r}{y i \text { ietm }}\right]}{\mathrm{SI}}$, avec yistr représentant le poids de coton graine du génotype $\mathbf{i}$ en condition de déficit hydrique, yietm le poids de coton graine du génotype $\mathbf{i}$ en condition hydrique optimale et SI l'intensité de stress.

Si DSI < 1, le génotype est tolérant au déficit hydrique et si DSI > 1 le génotype est sensible au déficit.
- Indice de tolérance au stress (STI), qui a été déterminé suivant la formule : $S T I=\frac{(\text { yistr })(\text { yietm })}{\mathrm{Y}^{2} \mathrm{etm}}$,

- Moyenne géométrique de la productivité (GMP), qui a été déterminé par la formule suivante : GMP $=\sqrt{(y s t r} *$ yetm $)$.

- Pertes relatives calculées suivant la formule : $\boldsymbol{P r} \boldsymbol{i} \boldsymbol{j}=\mathbf{1 0 0}\left(\frac{\text { Aijetm-Aijstr }}{\text { Aijetm }}\right)$; avec Aijstr $=$ valeur de la variété $\mathrm{i}$ pour la variable $\mathrm{j}$ en condition de déficit hydrique et Aijetm = valeur de la variété $\mathbf{i}$ pour la variable $\mathbf{j}$ en condition hydrique optimale.

\section{Traitement des données}

Les données collectées ont été saisies et traitées avec le tableur Excel et les analyses de la variance ont été effectuées avec le logiciel Genstat 2013. La corrélation de Pearson a été calculée pour déterminer les relations qui existent entre les différentes variables étudiées. Afin de déterminer les variétés tolérantes au stress hydrique, une régression de la moyenne géométrique de la productivité avec l'indice de sensibilité au stress a été effectué (Sezener et al., 2015) avec Excel 2013.

Tableau 1 : Caractéristiques physiques et hydriques du sol de la parcelle d'essai.

\begin{tabular}{|c|c|c|c|c|c|c|c|c|c|}
\hline \multirow[b]{2}{*}{$\begin{array}{c}\text { Horizon } \\
\text { (cm) }\end{array}$} & \multicolumn{5}{|c|}{ Proportion de chaque élément (\%) } & \multicolumn{2}{|c|}{ Humidité (\%) } & \multirow[b]{2}{*}{ Densité } & \multirow[b]{2}{*}{$\underset{(\mathbf{m m}) / \mathbf{c m}}{\mathbf{R U}}$} \\
\hline & Argile & Limon & $\begin{array}{c}\text { Limon } \\
\text { grossier }\end{array}$ & $\begin{array}{c}\text { Sable } \\
\text { fin }\end{array}$ & $\begin{array}{c}\text { Sable } \\
\text { grossier }\end{array}$ & PF 2,5 & PF 4,2 & & \\
\hline $0-20$ & 14,18 & 3,8 & 6,53 & 44,88 & 27,43 & 23,53 & 20,52 & 1,09 & 0,33 \\
\hline 20-100 & 28,85 & 4,8 & 1,02 & 35,37 & 25,85 & 28,84 & 26,25 & 1,8 & \\
\hline
\end{tabular}

NB : La Réserve Utile (RU) du sol a été déterminé suivant la formule suivante :

$\mathbf{R U}=\left(\mathbf{H}_{\mathrm{pf} 2,5^{-}} \mathbf{H}_{\mathrm{pf} 4,2}\right) \times \mathbf{Z} \times \mathbf{D a} ;$ où $\mathbf{H}_{\mathrm{pf} 2,5}=$ humidité à la capacité au champ $; \mathbf{H}_{\mathrm{pf} 4,2}=$ humidité au point de flétrissement $; \mathbf{Z}=$ profondeur de sol considérée en dm et $\mathbf{D a}=$ densité apparente du sol.

Tableau 2 : Quantité d'eau apportée suivant les différents régimes hydriques et les phases du cycle de développement du cotonnier.

\begin{tabular}{lccc}
\hline Phase du cycle & \multicolumn{2}{c}{ Quantité d'eau reçue $(\mathbf{m m})$} & $\begin{array}{c}\text { Réduction } \\
(\boldsymbol{\%})\end{array}$ \\
\hline Optimale & Déficit & $\mathbf{- 5 2}$ \\
Flomis-Floraison & 504,25 & 243,94 & $\mathbf{- 8 0}$ \\
Total (semis-récolte) & 462,65 & 91,27 & $\mathbf{- 6 5}$ \\
\hline
\end{tabular}



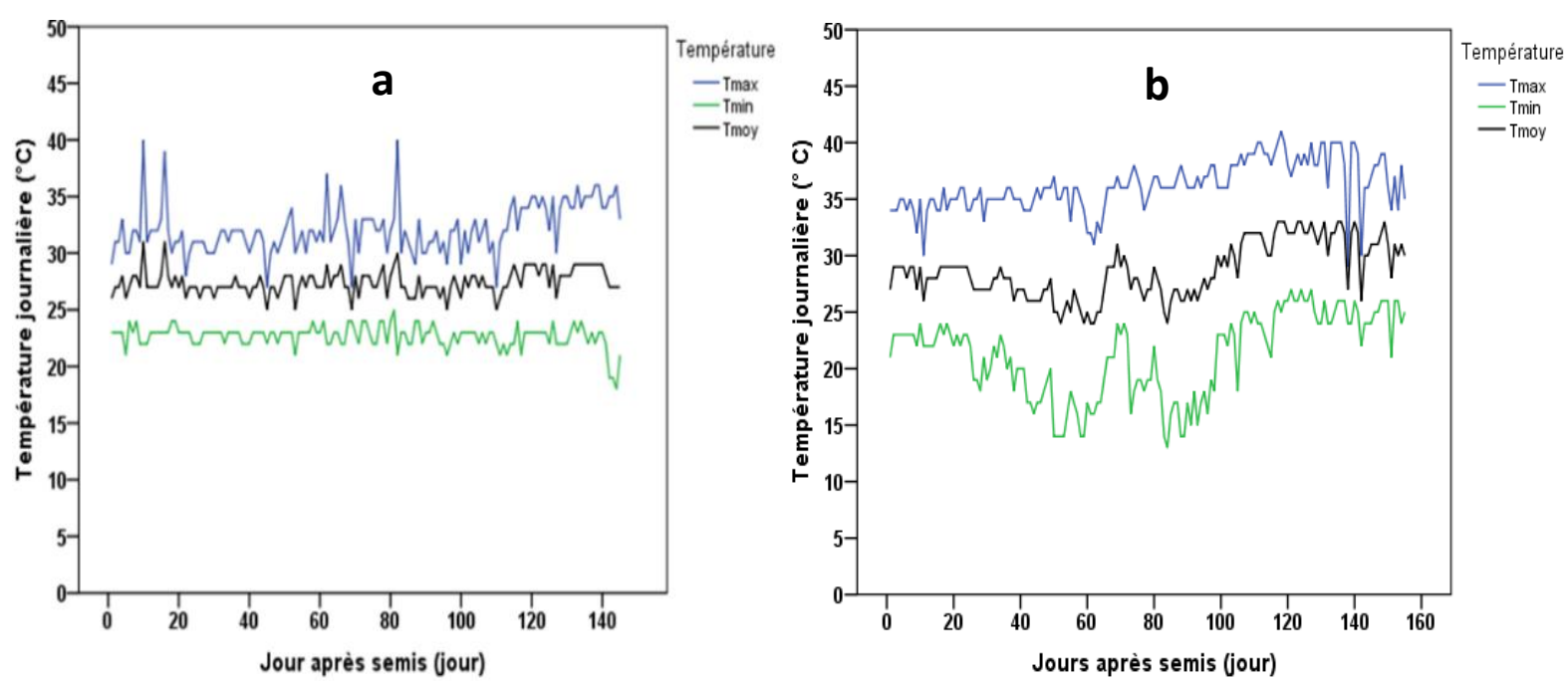

Figure 1 : Evolution des températures maximales, moyenne et minimale journalières sous abri à Kolokopé du semis à la récolte : $\mathbf{a}$ - condition hydrique optimale ; b- condition de déficit hydrique.

\section{RESULTATS}

\section{Description des variétés suivant leurs caractéristiques agronomiques, phénologiques et morphologiques \\ L'analyse de la variance a révélé un effet significatif des variétés sur toutes les variables étudiées aussi bien en condition hydrique optimale qu'en condition de déficit hydrique (Tableau 3).}

\section{Perte relative des caractères étudiés en condition de déficit hydrique comparativement à l'apport de l'eau en condition optimale.}

Les résultats ont montré que le déficit hydrique a réduit le rendement moyen en coton graine de $85 \%$, le PMC de $62 \%$, la précocité à la récolte de $24 \%$, la hauteur des plants à la floraison de $45 \%$, la hauteur des plants à la récolte de $35 \%$, le nombre de capsules par plant de $64 \%$ et le nombre de branches fructifères de $18 \%$ (Tableau 4). Par contre, le déficit hydrique a entrainé l'augmentation du nombre de branches végétatives de $44 \%$ et le retard de la floraison de $46 \%$.

La perte de rendement la plus élevée a été enregistrée chez la variété BRS Buriti (98\%) et la plus faible chez la variété X 148 (46\%).

En ce qui concerne la précocité de la récolte, les variétés se sont comportées différemment. Nous avons noté que $18 \%$ des variétés ont augmenté leur précocité de récolte, tandis que, le reste des variétés ont réduit leur précocité de récolte sous l'effet du stress. Les variétés ayant augmenté leur précocité de plus de 50\% ont été Oklahoma red, P 625-1, L 1561, BRS Araça et Guazuncho.

Corrélation entre les différents caractères mesurés et les indices de tolérance ou de sensibilité au stress calculé

Les résultats de corrélation ont montré que la relation entre les caractères mesurés d'une part, et d'autre part, entre les indices de stress et les caractères mesurés ont varié en fonction du régime hydrique.

En effet, en condition hydrique optimale, l'analyse de la matrice de corrélation (Tableau 5) a montré une liaison significative et positive entre le RDT et les caractères $\mathrm{HF}$, $\mathrm{HR}, \mathrm{BF}, \mathrm{CT}, \mathrm{R} 1 / \mathrm{RT}$; entre BF et les caractères $\mathrm{HF}$ et $\mathrm{HR}$; entre HF et HR.

En ce qui concerne les indices de stress et les caractères mesurés, les résultats ont montré des liaisons positives entre le DSI et les caractères CT et RDT ; entre les indices STI et le GMP et, les caractères BF, CT, R1/RT, PMC, RDT ; entre GMP et les caractères HF et HR. Par contre, une liaison négative a été trouvée entre DFL1 et les indices STI et GMP.

Les indices de stress STI et GMP ont été corrélés positivement mais, elles ont été corrélées négativement avec l'indice DSI. 
En condition de déficit hydrique, les résultats ont montré que le RDT a été lié positivement aux caractères $\mathrm{BF}, \mathrm{CT}, \mathrm{HF}, \mathrm{HR}$, R1/RT et PMC (Tableau 6). De même, il a été trouvé des liaisons positives entre $\mathrm{BF}$ et les caractères $\mathrm{HF}, \mathrm{HR}$ et R1/RT ; entre $\mathrm{BV}$ et les caractères DFL1 et HF; entre CT et les caractères HR, R1/RT et PMC; entre HF et HR.

Par ailleurs, des liaisons négatives ont été trouvées entre La DFL1 et les caractères R1/RT, RDT, CT, BF, HF et HR ; entre BV et $\mathrm{RDT}$; entre R1/RT et PMC.

Quant aux indices, le résultat a montré que le DSI a été lié positivement à DFL1 et négativement aux caractères $\mathrm{CT}, \mathrm{HR}, \mathrm{PMC}$ et RDT. Les indices STI et GMP ont été liés positivement aux caractères $\mathrm{BF}, \mathrm{CT}, \mathrm{HF}, \mathrm{HR}$, PRECO, PMC, RDT et négativement au caractère DFL1.

\section{Caractérisation des génotypes suivant les différents indices de stress}

Les indices de stress de chaque variété ont été consignés dans le Tableau 7. Les résultats ont montré que (i) le DSI a varié de 0,54 (X148) à 1,14 (F 379-15); (ii) le STI a varié de 0,11 (N'TA E 152) à 2,59 (AF 40113); (iii) le GMP a varié de 209,40 kg/ha (N'TA E 152) à 1020,74 (AF 401-13).
Le diagramme à double projections (biplot) avec le GMP en ordonné et le DSI en abscisse (Figure 2) a permis de classer les variétés en quatre groupes :

Groupe 1: caractérisé par les variétés tolérantes au déficit hydrique et ayant un fort potentiel de production. Ce groupe a été constitué de 31 variétés dont 9 du Togo, 9 des USA, 3 du Tchad, 2 du Mali et les autres du Bénin, du Brésil et de la Turquie.

Groupe 2 : caractérisé par les variétés sensibles au déficit hydrique mais ayant un fort potentiel de production. Ce groupe a été constitué de 34 variétés dont 24 du Togo, 4 du Brésil, 2 de la Côte d'Ivoire, 2 des USA et les autres du Tchad et de la Zambie.

Groupe 3 : caractérisé par les variétés sensibles au déficit hydrique et ayant un faible potentiel de production. Ce groupe a été constitué de 30 variétés dont 12 du Togo, 3 des USA, 3 de la Côte d'Ivoire et les autres du Burkina Faso, du Brésil, du Cameroun, du Costa Rica, du Mali et de la Thaïlande.

Groupe 4 : caractérisé par les variétés tolérantes au déficit hydrique mais ayant un faible potentiel de production. Ce groupe a été constitué de 23 variétés dont 3 du Togo, 2 des USA, 2 du Cameroun, 2 du Brésil, 2 du Mali, 2 de Costa Rica et les autres du Bénin, du Burkina Faso, de la Côte d'Ivoire, du Tchad et de l'Ouzbékistan.

Tableau 3 : Résultats statistiques des caractères étudiés.

\begin{tabular}{|c|c|c|c|c|c|c|c|c|c|c|c|}
\hline $\begin{array}{l}\text { Conditions } \\
\text { hydriques }\end{array}$ & VARIETES & BF & BV & $\mathbf{C B F}$ & CBV & CT & $\begin{array}{l}\text { HFL } \\
(\mathrm{cm})\end{array}$ & $\begin{array}{l}\text { HREC } \\
(\mathrm{cm})\end{array}$ & $\begin{array}{l}\text { DFL1 } \\
\text { (jas) }\end{array}$ & $\begin{array}{l}\text { R1/RT } \\
(\%)\end{array}$ & $\begin{array}{l}\text { RDT } \\
\text { (kg/ha) }\end{array}$ \\
\hline \multirow{6}{*}{ Optimale } & MIN & 10.04 & 1.30 & 5.92 & 1.17 & 8.27 & 75.50 & 75.50 & 59.35 & 15.53 & 632.00 \\
\hline & MAX & 16.71 & 4.91 & 17.82 & 10.65 & 25.93 & 137.90 & 137.90 & 76.01 & 63.07 & 2490.00 \\
\hline & MOY & 13.75 & 2.85 & 11.84 & 5.62 & 17.46 & 108.37 & 108.37 & 66.97 & 35.64 & 1616.05 \\
\hline & CV (\%) & 12.56 & 21.73 & 25.57 & 10.41 & 20.20 & 10.41 & 9.13 & 4.78 & 24.90 & 29.15 \\
\hline & $\mathbf{F}$ & 1.64 & 2.44 & 1.68 & 1.36 & 1.58 & 3.98 & 3.98 & 3.42 & 3.14 & 2.87 \\
\hline & $\mathbf{P}<\mathbf{f}$ & 0.00 & $<.001$ & $<.001$ & 0.03 & $<.001$ & $<.001$ & $<.001$ & $<.001$ & $<.001$ & $<.001$ \\
\hline \multirow{6}{*}{ Stress } & MIN & 8.19 & 2.57 & 0.79 & 0.01 & 1.16 & 42.46 & 46.38 & 91.71 & 0.00 & 28.30 \\
\hline & MAX & 14.22 & 6.73 & 8.77 & 3.53 & 15.12 & 68.57 & 81.76 & 114.50 & 66.17 & 449.10 \\
\hline & MOY & 11.27 & 4.01 & 3.50 & 1.69 & 6.06 & 59.47 & 69.51 & 97.82 & 12.00 & 249.35 \\
\hline & $\mathrm{CV}$ & 9.97 & 18.2 & 31.85 & 51.38 & 28.27 & 8.68 & 8.9 & 2.78 & 39.96 & 31.5 \\
\hline & $\mathbf{F}$ & 3.01 & 3.64 & 2.6 & 2.31 & 3.47 & 2.85 & 3.04 & 5.55 & 4.48 & 3.76 \\
\hline & $\mathbf{P}<\mathbf{f}$ & $<.001$ & $<.001$ & $<.001$ & $<.001$ & $<.001$ & $<.001$ & $<.001$ & $<.001$ & $<.001$ & $<.001$ \\
\hline
\end{tabular}

NB : BF : Nombre de branches fructifères ; BV : Nombre de branches végétatives ; CBF : Nombre de capsules portées par les BF ; CBV : Nombre de capsules portées par les BV ; CT : Nombre de capsules totales portées par le plant ; HF : Hauteur des plants à la floraison; HR : Hauteur des plants à la récolte ; DFL1 : Date de la floraison moyenne ; R1/RT : Précocité de la récolte ; PMC : Poids moyen capsulaire ; RDT : Rendement en coton graine. 
Tableau 4 : Perte relative des valeurs des caractères étudiés en condition de déficit par rapport à la condition hydrique optimale.

\begin{tabular}{cccccccccccc}
\hline \multirow{2}{*}{ Perte (\%) } & \multicolumn{10}{c}{ Variables } \\
\cline { 2 - 12 } & BF & BV & CBF & CBV & CT & D1FL & HF & HR & PREC & PMC & RDT \\
\hline Min. & -12 & -116 & 16 & -65 & 5 & -60 & 31 & 16 & -216 & 28 & 46 \\
Max. & 40 & 21 & 90 & 100 & 90 & -23 & 57 & 53 & 114 & 76 & 98 \\
Moy. Pondérale & 18 & -44 & 70 & 65 & 64 & -46 & 45 & 35 & 23 & 62 & 84 \\
\hline
\end{tabular}

Tableau 5 : Matrice de corrélation entre les indices de stress et les caractères mesurés en condition hydrique optimale.

\begin{tabular}{|c|c|c|c|c|c|c|c|c|c|c|c|c|}
\hline Variables & BF & BV & CT & DFL1 & $\mathbf{H F}$ & HR & R1/RT & PMC & RDT & DSI & STI & GMP \\
\hline$\overline{B F}$ & 1 & & & & & & & & & & & \\
\hline BV & .087 & 1 & & & & & & & & & & \\
\hline CT & .115 & $.193^{*}$ & 1 & & & & & & & & & \\
\hline DFL1 & -.158 & .132 & $-.367^{* *}$ & 1 & & & & & & & & \\
\hline HF & $.477^{* *}$ & $.269^{* * *}$ & .033 & .076 & 1 & & & & & & & \\
\hline HR & $.477^{* *}$ & $.269^{* * *}$ & .033 & .076 & $1.00^{* *}$ & 1 & & & & & & \\
\hline R1/RT & .072 & -.135 & .156 & $-.676^{* *}$ & -.114 & -.114 & 1 & & & & & \\
\hline PMC & .043 & -.043 & -.021 & -.017 & .007 & .007 & .039 & 1 & & & & \\
\hline RDT & $.267^{* *}$ & .118 & $.371^{* * *}$ & $-.431^{* *}$ & $.312^{* * *}$ & $.312^{* * *}$ & $.372^{* * *}$ & $.194^{*}$ & 1 & & & \\
\hline DSI & .047 & .113 & $.211^{*}$ & -.023 & .177 & .177 & .085 & .110 & $.377^{* *}$ & 1 & & \\
\hline STI & $.279^{* *}$ & .040 & $.213^{*}$ & $-.395^{* *}$ & .175 & .175 & $.226^{*}$ & $.206^{*}$ & $.674^{* *}$ & $-.188^{*}$ & 1 & \\
\hline GMP & $.306^{* *}$ & .044 & $.224^{*}$ & $-.378^{* *}$ & $.216^{*}$ & $.216^{*}$ & $.209^{*}$ & $.217^{*}$ & $.683^{* *}$ & $-.216^{*}$ & $.981^{* *}$ & 1 \\
\hline
\end{tabular}

Tableau 6 : Matrice de corrélation entre les indices de stress et les caractères mesurés en condition de déficit hydrique.

\begin{tabular}{|c|c|c|c|c|c|c|c|c|c|c|c|c|}
\hline & BF & BV & CT & DFL1 & HF & HR & R1/RT & PMC & RDT & DSI & STI & GMP \\
\hline$\overline{B F}$ & 1 & & & & & & & & & & & \\
\hline BV & -.136 & 1 & & & & & & & & & & \\
\hline CT & .141 & -.039 & 1 & & & & & & & & & \\
\hline DFL1 &.$- .333^{* * *}$ & $.273^{* * *}$ & $-.486^{* *}$ & 1 & & & & & & & & \\
\hline HF & $.465^{* *}$ & $.265^{* *}$ & .105 & $-.251^{* * *}$ & 1 & & & & & & & \\
\hline HR & $.561^{* *}$ & .172 & $.193^{*}$ & $-.326^{* *}$ & $.845^{* *}$ & 1 & & & & & & \\
\hline R1/RT &. .333 $^{* *}$ & $-.390^{* *}$ & $.408^{* * *}$ & $-.766^{* * *}$ & .015 & .120 & 1 & & & & & \\
\hline PMC & -.149 & .167 & $.201^{*}$ & .009 & .039 & -.014 & $-.194^{*}$ & 1 & & & & \\
\hline RDT & .161 & -.119 & $.693^{* *}$ & $-.579^{* * *}$ & $.256^{* * *}$ & $.295^{* *}$ & $.382^{* * *}$ & $.327^{* *}$ & 1 & & & \\
\hline DSI & -.052 & -.016 & $-.458^{* *}$ & $.233^{*}$ & -.142 & $-.221^{*}$ & -.100 & $-.254^{* * *}$ & $-.612^{* * *}$ & 1 & & \\
\hline STI & $.259^{* *}$ & -.171 & $.464^{* *}$ & $-.353^{* *}$ & $.218^{*}$ & $.199^{*}$ & $.266^{* * *}$ & $.293^{* *}$ & $.706^{* *}$ & $-.188^{*}$ & 1 & \\
\hline GMP & $.262^{* *}$ & -.178 & $.472^{* * *}$ & $-.390^{* *}$ & $.272^{* *}$ & $.253^{* *}$ & $.260^{* * *}$ & $.324^{* *}$ & $.720^{* *}$ & $-.216^{*}$ & $.981^{* *}$ & 1 \\
\hline
\end{tabular}


Tableau 7 : Moyenne des rendements en coton graine sous les deux régimes hydriques et les indices de tolérance au stress suivant les variétés.

\begin{tabular}{|c|c|c|c|c|c|c|c|}
\hline \multirow[t]{2}{*}{ Groupes } & \multirow[t]{2}{*}{ VARIETES } & \multirow[t]{2}{*}{ Origines } & \multicolumn{2}{|c|}{$\begin{array}{l}\text { Rendement coton } \\
\text { graine (kg/ha) }\end{array}$} & \multirow[t]{2}{*}{ DSI } & \multirow[t]{2}{*}{ STI } & \multirow[t]{2}{*}{ GMP } \\
\hline & & & Déficit & Irrigué & & & \\
\hline 1 & AF 401-13 & TOGO & 449.1 & 2320 & 0.95 & 2.59 & 1020.74 \\
\hline 1 & Deltapine 5690 & USA & 391.8 & 2313 & 0.98 & 2.25 & 951.96 \\
\hline 1 & NTA 93-15 & MALI & 431 & 1998 & 0.93 & 2.14 & 927.98 \\
\hline 1 & COCKER 4104 & USA & 410.2 & 2048 & 0.95 & 2.08 & 916.56 \\
\hline 1 & $\begin{array}{l}\text { STONEVILLE } \\
\text { FREGO }\end{array}$ & USA & 440.4 & 1866 & 0.9 & 2.04 & 906.52 \\
\hline 1 & В 163 & CI & 327.4 & 2016 & 0.99 & 1.64 & 812.43 \\
\hline 1 & COCKER 320 & USA & 349.2 & 1887 & 0.96 & 1.64 & 811.75 \\
\hline 1 & COCKER 4107 & USA & 393.8 & 1673 & 0.9 & 1.63 & 811.68 \\
\hline 1 & STONEVILLE 506 & USA & 362.6 & 1793 & 0.94 & 1.61 & 806.31 \\
\hline 1 & A 51 & TCHAD & 415.7 & 1556 & 0.87 & 1.61 & 804.26 \\
\hline 1 & D388-8 & TOGO & 353.2 & 1764 & 0.95 & 1.55 & 789.33 \\
\hline 1 & K 615-10 & TOGO & 381.6 & 1614 & 0.9 & 1.53 & 784.79 \\
\hline 1 & BRS 293 & BRESIL & 336.7 & 1787 & 0.96 & 1.49 & 775.68 \\
\hline 1 & $\begin{array}{l}\text { MACNAIR 511-D388- } \\
8\end{array}$ & TOGO & 366.7 & 1639 & 0.92 & 1.49 & 775.26 \\
\hline 1 & A $209-10$ & TOGO & 316.2 & 1866 & 0.98 & 1.46 & 768.13 \\
\hline 1 & K 523-6 & TOGO & 349.6 & 1638 & 0.93 & 1.42 & 756.73 \\
\hline 1 & P $625-1$ & BENIN & 344.5 & 1635 & 0.93 & 1.4 & 750.5 \\
\hline 1 & X 260 & TCHAD & 379.3 & 1261 & 0.83 & 1.19 & 691.59 \\
\hline 1 & Н 226 & TOGO & 281.7 & 1692 & 0.99 & 1.18 & 690.39 \\
\hline 1 & SIOKRA L 22 & CIRAD & 312.1 & 1517 & 0.94 & 1.17 & 688.08 \\
\hline 1 & NTA 88-6 & MALI & 312.2 & 1481 & 0.93 & 1.15 & 679.98 \\
\hline 1 & TAMCOTSP21-5 & USA & 284.5 & 1602 & 0.97 & 1.13 & 675.11 \\
\hline 1 & J 129A & TOGO & 307 & 1471 & 0.94 & 1.12 & 672.01 \\
\hline 1 & MACNAIR 511 & USA & 318.8 & 1393 & 0.91 & 1.1 & 666.4 \\
\hline 1 & SRT1 & CIRAD & 323.2 & 1363 & 0.9 & 1.09 & 663.72 \\
\hline 1 & NAZILI & TURKI & 288.3 & 1527 & 0.96 & 1.09 & 663.5 \\
\hline 1 & STONE 907 & USA & 284.8 & 1532 & 0.96 & 1.08 & 660.54 \\
\hline 1 & A 26 & TCHAD & 281.6 & 1532 & 0.97 & 1.07 & 656.82 \\
\hline 1 & Z 538-10 & TOGO & 310.8 & 1361 & 0.91 & 1.05 & 650.38 \\
\hline 2 & AJ 275 (1.2.6.7.14) & TOGO & 386.5 & 2449 & 1 & 2.35 & 972.9 \\
\hline 2 & AF 436-3 & TOGO & 324.4 & 2158 & 1 & 1.74 & 836.69 \\
\hline 2 & AJ $275-2$ & TOGO & 316.7 & 2119 & 1.01 & 1.67 & 819.2 \\
\hline 2 & AK 688-2 & TOGO & 314.9 & 2129 & 1.01 & 1.66 & 818.79 \\
\hline 2 & AK 538-2 & TOGO & 309.3 & 2096 & 1.01 & 1.61 & 805.17 \\
\hline
\end{tabular}




\begin{tabular}{|c|c|c|c|c|c|c|c|}
\hline 2 & H 406-7 FREGO & USA & 290.2 & 2198 & 1.03 & 1.58 & 798.66 \\
\hline 2 & SIND $60 * \mathrm{~F} 280$ & TOGO & 275.4 & 2264 & 1.04 & 1.55 & 789.62 \\
\hline 2 & BRS 286 & BRESIL & 287.4 & 2143 & 1.02 & 1.53 & 784.79 \\
\hline 2 & F490-7 & TOGO & 294.6 & 2055 & 1.01 & 1.5 & 778.08 \\
\hline 2 & O 480-6 & TOGO & 289.7 & 2049 & 1.02 & 1.47 & 770.45 \\
\hline 2 & AF $400-10$ & TOGO & 282.3 & 2081 & 1.02 & 1.46 & 766.46 \\
\hline 2 & S 295 & TCHAD & 277.4 & 2087 & 1.03 & 1.44 & 760.88 \\
\hline 2 & K 542-7 & TOGO & 241.1 & 2399 & 1.06 & 1.44 & 760.53 \\
\hline 2 & STAM 279 A & TOGO & 289.7 & 1952 & 1.01 & 1.4 & 751.99 \\
\hline 2 & STAM 190 & TOGO & 227.1 & 2407 & 1.07 & 1.36 & 739.34 \\
\hline 2 & STAM 82 & TOGO & 287.4 & 1846 & 1 & 1.32 & 728.38 \\
\hline 2 & AK 705-2 & TOGO & 277.2 & 1888 & 1.01 & 1.3 & 723.43 \\
\hline 2 & FREGO_VAR & USA & 234.9 & 2090 & 1.05 & 1.22 & 700.67 \\
\hline 2 & AK 605-5 & TOGO & 240.8 & 2024 & 1.04 & 1.21 & 698.13 \\
\hline 2 & AK 737-7 & TOGO & 250.8 & 1928 & 1.03 & 1.2 & 695.37 \\
\hline 2 & STAM 59 & TOGO & 204.4 & 2327 & 1.08 & 1.18 & 689.67 \\
\hline 2 & BRS AROERA & BRESIL & 187.5 & 2473 & 1.09 & 1.15 & 680.95 \\
\hline 2 & AF 425-9 & TOGO & 250.1 & 1853 & 1.02 & 1.15 & 680.76 \\
\hline 2 & AF 577-13 & TOGO & 215.2 & 2126 & 1.06 & 1.14 & 676.4 \\
\hline 2 & AK 693-10 & TOGO & 264.8 & 1725 & 1 & 1.13 & 675.86 \\
\hline 2 & BRS ARARIPA & BRESIL & 264.4 & 1721 & 1 & 1.13 & 674.56 \\
\hline 2 & G 319-16 & CI & 208.4 & 2167 & 1.07 & 1.12 & 672.01 \\
\hline 2 & BRS CERIDO & BRESIL & 223 & 2009 & 1.05 & 1.11 & 669.33 \\
\hline 2 & L46B & TOGO & 224.9 & 1987 & 1.05 & 1.11 & 668.49 \\
\hline 2 & STAMF & TOGO & 251.4 & 1774 & 1.01 & 1.11 & 667.82 \\
\hline 2 & J 292 & TOGO & 227.5 & 1883 & 1.04 & 1.06 & 654.51 \\
\hline 2 & AJ 179-11 & TOGO & 206.7 & 2062 & 1.06 & 1.06 & 652.85 \\
\hline 2 & G 165 & CI & 184.7 & 2269 & 1.09 & 1.04 & 647.37 \\
\hline 2 & CD14 & Zambie & 202.2 & 2028 & 1.06 & 1.02 & 640.36 \\
\hline 3 & BRS CEDRO & BRESIL & 242.6 & 1587 & 1 & 0.96 & 620.49 \\
\hline 3 & N'TAL 88 & MALI & 197.1 & 1901 & 1.06 & 0.93 & 612.12 \\
\hline 3 & ALLEN 333-57 & TOGO & 188.1 & 1865 & 1.06 & 0.87 & 592.29 \\
\hline 3 & STONEVILLE & USA & 211.4 & 1654 & 1.03 & 0.87 & 591.32 \\
\hline 3 & M 307-10 GL & CI & 202.7 & 1704 & 1.04 & 0.86 & 587.71 \\
\hline 3 & STAM 129A & TOGO & 212.8 & 1554 & 1.02 & 0.82 & 575.06 \\
\hline 3 & BRS JATOBA & BRESIL & 183 & 1776 & 1.06 & 0.81 & 570.09 \\
\hline 3 & IRCT CR 80 & CI & 154.7 & 2083 & 1.09 & 0.8 & 567.66 \\
\hline 3 & Deltaprine SL FREGO & COSTA-RICA & 119.9 & 2490 & 1.13 & 0.74 & 546.4 \\
\hline 3 & W 766A & USA & 167.2 & 1612 & 1.06 & 0.67 & 519.16 \\
\hline 3 & ACAJOU & $\mathrm{RCI}$ & 182.6 & 1412 & 1.03 & 0.64 & 507.77 \\
\hline 3 & STAM 86 & TOGO & 164.9 & 1512 & 1.05 & 0.62 & 499.33 \\
\hline
\end{tabular}




\begin{tabular}{|c|c|c|c|c|c|c|c|}
\hline 3 & L212-5 & TOGO & 145.6 & 1613 & 1.08 & 0.58 & 484.62 \\
\hline 3 & FK 37 & TOGO & 160.1 & 1450 & 1.05 & 0.58 & 481.81 \\
\hline 3 & K 592-7 & $\mathrm{BF}$ & 156.1 & 1447 & 1.05 & 0.56 & 475.26 \\
\hline 3 & X 442A & TOGO & 161.5 & 1166 & 1.02 & 0.47 & 433.95 \\
\hline 3 & AK 587-3 & $\mathrm{CI}$ & 154.6 & 1215 & 1.03 & 0.47 & 433.4 \\
\hline 3 & L $156-3$ & TOGO & 130.4 & 1384 & 1.07 & 0.45 & 424.82 \\
\hline 3 & IRMA 1178 & TOGO & 99.3 & 1463 & 1.1 & 0.36 & 381.15 \\
\hline 3 & $\mathrm{LC} 1$ & $\mathrm{CR}$ & 98 & 1412 & 1.1 & 0.34 & 371.99 \\
\hline 3 & DPL 90 & TOGO* & 124.5 & 998 & 1.03 & 0.31 & 352.49 \\
\hline 3 & PRONTO OKRA & USA & 91.3 & 1127 & 1.09 & 0.26 & 320.77 \\
\hline 3 & STAM 45E & CIRAD & 91.7 & 1106 & 1.08 & 0.25 & 318.47 \\
\hline 3 & AK 779-8 & TOGO & 72.6 & 832 & 1.08 & 0.15 & 245.77 \\
\hline 3 & F $379-15$ & TOGO & 44.8 & 1245 & 1.14 & 0.14 & 236.17 \\
\hline 3 & SRISRAM RONG 60 & TOGO & 67 & 813 & 1.09 & 0.14 & 233.39 \\
\hline 3 & N'TA E 152 & Thaillande & 40.6 & 1080 & 1.14 & 0.11 & 209.4 \\
\hline 4 & IRCT CR 10 & MALI & 255.5 & 1492 & 0.98 & 0.95 & 617.42 \\
\hline 4 & Deltapine & COSTA-RICA & 342.5 & 1067 & 0.8 & 0.91 & 604.52 \\
\hline 4 & FK 64 & USA & 322 & 1134 & 0.85 & 0.91 & 604.27 \\
\hline 4 & HG 151-5 & $\mathrm{BF}$ & 250.6 & 1384 & 0.97 & 0.86 & 588.92 \\
\hline 4 & J 394-7 & CIRAD & 246.8 & 1372 & 0.97 & 0.84 & 581.9 \\
\hline 4 & U 633 & TOGO & 374.8 & 857 & 0.67 & 0.8 & 566.75 \\
\hline 4 & GUAZUNCHO & COSTA-RICA & 363.6 & 878 & 0.69 & 0.79 & 565.01 \\
\hline 4 & K 636-10 & ARGENTINE & 329.4 & 968 & 0.78 & 0.79 & 564.68 \\
\hline 4 & W 885 & TOGO & 247 & 1267 & 0.95 & 0.78 & 559.42 \\
\hline 4 & AJ 237-9 & CAMEROUN & 233 & 1281 & 0.97 & 0.74 & 546.33 \\
\hline 4 & BRS SAFIRA & TOGO & 269.5 & 1033 & 0.87 & 0.69 & 527.63 \\
\hline 4 & NTA 93-2 & BRESIL & 219.9 & 1204 & 0.97 & 0.66 & 514.55 \\
\hline 4 & PAVLIKENI & MALI & 235.1 & 1073 & 0.92 & 0.63 & 502.26 \\
\hline 4 & BRS ARACA & & 285.1 & 845 & 0.78 & 0.6 & 490.83 \\
\hline 4 & X 148 & BRESIL & 347.7 & 641 & 0.54 & 0.55 & 472.1 \\
\hline 4 & TASKENT 6 & TCHAD & 185 & 1001 & 0.96 & 0.46 & 430.33 \\
\hline 4 & P 746-1 & Pakistan & 205.8 & 860 & 0.9 & 0.44 & 420.7 \\
\hline 4 & ISA 268 & BENIN & 150.3 & 903 & 0.99 & 0.34 & 368.4 \\
\hline 4 & NIAB 78 & CI & 138.3 & 864 & 0.99 & 0.3 & 345.67 \\
\hline 4 & IRMA 910 & Uzbekistan & 163.1 & 639 & 0.88 & 0.26 & 322.83 \\
\hline 4 & NTA 88-6 (2017) & $\mathrm{CR}$ & 121.5 & 632 & 0.96 & 0.19 & 277.11 \\
\hline 4 & OKLOHOMA RED & MALI & 110.4 & 674 & 0.99 & 0.18 & 272.78 \\
\hline
\end{tabular}




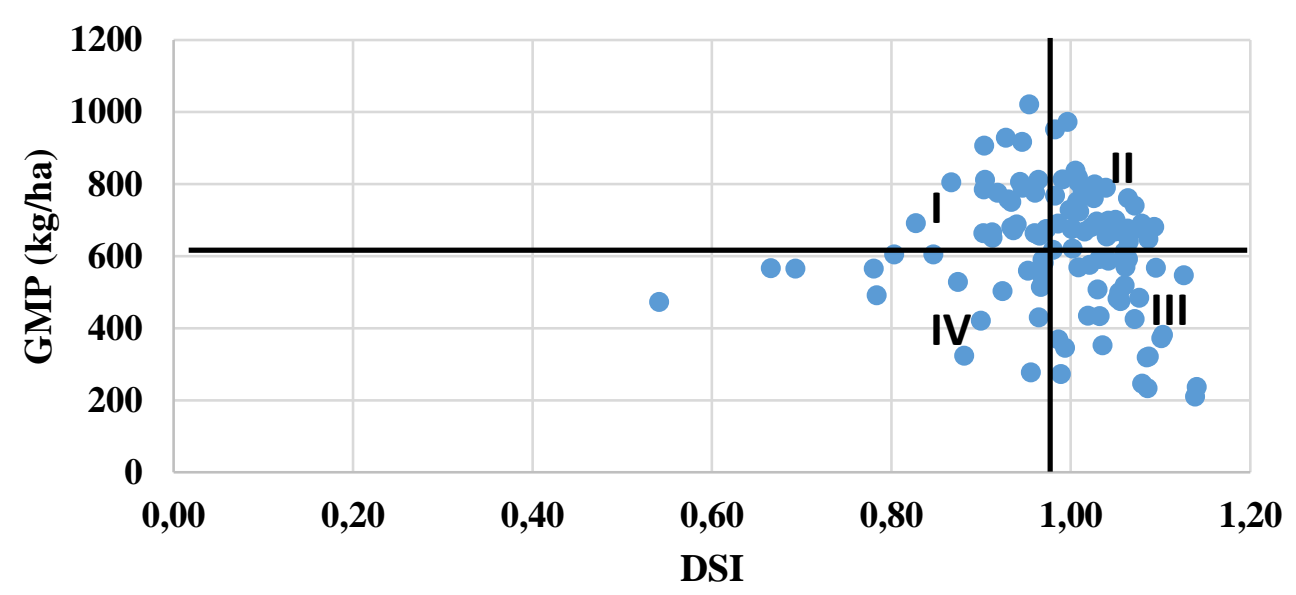

Figure 2 : Biplot entre la Moyenne Géométrique de la production (GMP) et l'indice de sensibilité au stress hydrique (DSI) des variétés de cotonnier testées.

\section{DISCUSSION}

L'effet significatif des deux régimes hydriques sur tous les caractères étudiés des variétés montre qu'il existe une grande variabilité au sein des accessions étudiées pour tous ces caractères. En effet, les différences significatives observées entre les variétés en condition de déficit hydrique montrent qu'il existe une variabilité de réponse au déficit hydrique qu'on peut exploiter. Cette grande variabilité s'explique en partie par les conditions de culture pour lesquelles ces variétés ont été sélectionnées. Les variétés des pays africains et d'autres pays moins développés sont créées pour la culture pluviale et la récolte manuelle tandis que, la plupart des variétés des pays développés sont créées pour la culture irriguée et les récolte mécanique.

Le déficit hydrique a entrainé une perte importante de la production due à la perte des organes fructifères, du nombre de capsules et du poids moyen des capsules. De nombreux études ont montré que le déficit hydrique entraine chez le cotonnier, la perte de la production en quantité et en qualité (Pettigrew, 2004 ; Zare et al., 2014 ; Sezener et al., 2015). Des pertes importantes à modérées de rendement dues au déficit hydrique sur d'autres spéculations telles que le maïs, le riz, le sorgho, le niébé, l'arachide, l'orge, le blé et la patate douce ont été trouvées par de nombreux auteurs (Al-Khayri et al., 2014 ; Mahamat et al., 2014 ; Akata, 2018). En considérant les pertes relatives de rendement en coton graine, nous avons constaté que les variétés à faible potentiel de production (X 148, U 633) sont plus stables. Leur perte de rendement en situation de stress reste inférieure à celle des variétés à fort potentiel de rendement. Cependant, quand bien même certaines variétés à fort potentiel de rendement ont des pertes de rendement très élevées, leur production en condition de déficit reste toujours supérieure à celles des variétés stables. C'est le cas des variétés F401-13, Deltapine 5690, NTA 93-15, COCKER 4104 et STONEVILLE FREGO et AJ 275 (1.2.6.7.14).

Sur le plan phénologique, la réaction au déficit varie d'une variété à une autre. Il y a eu des variétés qui ont allongé leur cycle pour permettre aux capsules de se former lorsque l'eau sera disponible tandis que, d'autres ont raccourci leur cycle de développement pour éviter le déficit. Akata (2018) a trouvé que l'application d'un déficit pré-floral à une accession de sorgho Ouest africain a induit les mêmes mécanismes de réponse que ceux observés sur cotonnier dans cette étude.

Sous les deux régimes hydriques, le rendement en coton graine est lié positivement 
à la charge capsulaire, à la taille des plants et au poids moyen capsulaire. La liaison positive entre le rendement et le nombre de capsules par plants montre que la rétention des capsules est un facteur déterminant à l'amélioration du rendement en condition de déficit hydrique ou non (Rahman et al., 2008). De même, la liaison positive entre le rendement et le PMC montre que sous déficit hydrique, les variétés qui arrivent à accumuler plus d'hydrate de carbone donnent les capsules les plus grosses et par ricochet de bons rendements. La meilleure accumulation d'hydrate de carbone en condition de déficit n'est possible que si l'activité photosynthétique est intense (AlKayri et al., 2014 ; Khan et al., 2018). Donc, les variétés ayant eu des poids moyens capsulaires élevés auraient de meilleures activités photosynthétiques.

Le rendement en coton graine a été négativement lié à la date d'apparition de la première fleur et positivement avec la précocité de la récolte sous les deux régimes. De nombreux auteurs ont montré que la précocité fait partie des mécanismes de résistance (par évitement) au déficit hydrique mis en jeu par les plants de cotonnier (Al-Kayri et al., 2014 ; Khan et al., 2018).

La forte corrélation positive entre le rendement et les indices STI et GMP en condition hydrique optimale et déficitaire montre que les variétés les plus productives en condition optimale sont celles qui donnent des rendements en coton graine élevés en condition de déficit. Ce résultat est similaire à celui trouvé par Sezener et al. (2015). Ces résultats montrent que le rendement en coton graine reste un bon critère de sélection en condition de déficit hydrique comme en condition hydrique optimale.

La corrélation négative entre le DSI et les composantes du rendement (CT et PMC) en condition de déficit montre que ces deux caractéristiques peuvent être utilisées comme des critères pour la sélection des variétés du cotonnier résistantes au déficit hydrique.

La structuration de la variabilité au déficit hydrique au sein des variétés étudiées nous a permis d'identifier le groupe de variétés tolérantes avec un fort potentiel de production. Ce groupe est constitué majoritairement (55\%) des variétés créées en Afrique de l'Ouest (Bénin, Côte d'Ivoire, Mali et Togo). En effet, dans ces pays, la sélection est faite exclusivement en condition pluviale, marquée parfois par des poches de sécheresse. En sélectionnant le génotype le plus productif dans de telles conditions, on a la chance de sélectionner indirectement les génotypes résistants au déficit hydrique. C'est ce qui explique que ce groupe soit constitué majoritairement des variétés de l'Afrique de l'Ouest. En dehors des variétés africaines, ce groupe est constitué des variétés Américaines dont le fond génétique à une très bonne précocité (Dessauw et Hau, 1997). Les variétés de ce groupe peuvent être utilisées pour l'amélioration de la tolérance au déficit hydrique au Togo et en Afrique de l'Ouest.

\section{Conclusion}

L'un des facteurs limitants de la production cotonnière en Afrique de l'Ouest et plus particulièrement au Togo est la sécheresse. Afin de minimiser son impact négatif sur la production cotonnière $\mathrm{du}$ Togo, nous avons entrepris d'identifier parmi les variétés de cotonnier (G. hirsutum) disponibles au Togo, celles qui sont tolérantes au déficit hydrique. À l'issu de cette étude, il a été noté la présence d'une grande variabilité au déficit hydrique au sein des variétés testées. Les résultats ont révélé que, le déficit hydrique a entrainé une réduction du rendement en coton graine de $65 \%$. Cette réduction est liée à la baisse du nombre de capsules par plant et au poids moyen capsulaire. Enfin, nous avons identifié les variétés tolérantes à fort potentiel de production (groupe 1); les variétés sensibles à fort potentiel de production (groupe2); les variétés sensibles à faible potentiel de production (groupe 3) et des variétés résistantes à faible potentiel de production (groupe4). Les résultats de cette étude constituent une avancée pour une meilleure exploitation des ressources génétiques du cotonnier disponibles au Togo. Les variétés résistantes à fort potentiel de production 
identifiées seront une source importante de géniteurs pour l'amélioration des variétés vulgarisées au Togo à la résistance au déficit hydrique.

\section{CONFLIT D'INTERETS}

Les auteurs déclarent qu'ils n'ont aucun conflit d'intérêts.

\section{CONTRIBUTIONS DES AUTEURS}

Tous les auteurs ont participé à la rédaction de cet article mais à des degrés divers : KZK rédaction des protocoles, NG collectes des données, KPA rédaction de l'article et MA et KT lecture.

\section{REMERCIEMENTS}

Nos remerciements vont à la Nouvelle société cotonnière du Togo et à l'Institut Togolais de recherche Agronomique pour le financement de ce projet de recherche.

\section{REFERENCES}

Adewi E, Badameli KMS, Dubreuil V. 2010. Evolution des saisons des pluies potentiellement utiles au Togo de 1950 à 2000. Climatologie, 7: 89-107. DOI: 10.4267/climatologie.489

Akata EA. 2018. Analyse de la diversité génétique du sorgho [Sorghum bicolor (L.) Moench] et identification d'accessions prometteuses pour l'amélioration de l'adaptation à la sécheresse en Afrique de l'Ouest. Thèse de Doctorat, Université Chekh Anta Diop, Dakar, 103 p.

Al-Khayri JM, Rauf S, Zaharieva M, Monneveux P, Khalil F. 2015. Breeding strategies to enhance Drought Tolerance in crops. In Advance in plant breeding strategies, Agronomic, Abiotic and Biotic Stress Traits, Al-KhayriShri JM, Jain M, Johnson DV (eds). Springer: Switzerland; 1-70. DOI : 10.13140/2.1.2343.9682

Bakayoko K. 2013. L'importance et l'avenir du coton en Afrique de l'Ouest : cas du Mali. Thèse de Doctorat, Université de Grenoble, Grenoble, 412 p.
Bokobana A, Toundou O, Odah K, Dossou KSS, Tozo K. 2019. Enhancement of proline content and antioxidant enzyme activities induced by drought stress in maize (Zea mays L.) by application of compost. Int. J. Biol. Chem. Sci., 13(7): 2978-2990. DOI : 10.4314/ijbcs.v13i7.1

Dagnelie P.

2012. Principes d'Expérimentation : Planification des Expériences et Analyse de leurs Résultats (ed). Presses Agronomiques: Gembloux.

Dessauw D, Hau B. 1997. Manuel pour l'Amélioration $d u$ Cotonnier (ed). CIRAD-Agritrop : Montpellier.

Djaman K, Sharma V, Rudnick DR, Koudahe K, Irmak S, Amouzou KA, Sogbedji JM. 2017. Spatial and Temporal Variation in Precipitation in Togo. Int. J. Hydro., 1(4): 00019. DOI: 10.15406/ijh.2017.01.00019

Gadedjisso-Tossou A, Adjegan K, Kablan AKM. 2020. Rainfall and Temperature Trend Analysis by Mann-Kendall Test and Significance for Rainfed Cereal Yields in Northern Togo. Science, 2(74): 1-23. DOI: $10.3390 /$ sci2040074

Gnofam N, Tozo K, Bonfoh B, Akantetou KP, Kolani L, Amouzouvi K. 2014. Effet d'un déficit hydrique sur certains paramètres morphologiques, physiologiques et le rendement chez le cotonnier (Gossypium hirsutum L. cv STAM 129A) cultivé au Togo. Agro. Afri., 26(2): 113-125.

Karademir C, Karademir E, Ekinci R, Berekatogu K. 2011. Yield and fiber quality properties of cotton (Gossypium hirsutum L.) under water stress and nonstress conditions. African Journal of Biotechnology, 10(59): 12575-12583. DOI: $10.5897 / A J B 11.1118$

Khan A, Pan X, Najeeb U, Tan DKY, Fahad S, Zahoor R, Luo H. 2018. Coping with drought: stress and adaptive mechanisms, and management through cultural and molecular alternatives in cotton as vital constituents for plant stress resilience and fitness. Biol. Res., 51(1): 1-17. DOI: https://doi.org/10.1186/s40659-0180198-z 
Koffi KZ, Aziadekey M, Akantetou KP, Gnofam N, Ayeva B, Kilimou P, Bonfoh B. 2017. Evaluation de l'incidence des attaques des piqueurs-suceurs sur huit variétés de cotonnier (Gossypium hirsutum) au Togo. J. Rech. Sci., 19(2): 247-257.

Koffi KZ, Gnofam N, Akantetou KP, Ayeva B, Bonfoh B, Koinzi PI, Ogou A, Mawussi G, Aziadekey M. 2019. Évaluation du progrès génétique des variétés de cotonnier (Gossypium hirsutum) créées et cultivées au Togo de 1981 à 2017. BRAB, 12(S3): 74-80.

Koudahe K, Djaman K, Kayode JA, Awokola SO, Adebola AA. 2018. Impact of Climate Variability on Crop Yields in Southern Togo. Environ. Pollut. Clim. Chang., 2(1): 1-9. DOI: 10.4172/2573458X.1000.148

Lacape J-M, Loison R, Foncéka D. 2015. Amélioration de l'adaptation des cultures à la sécheresse en zone de savane africaine. In Changement Climatique et Agriculture $d u$ Monde, Torquebiau E (ed). CIRAD : Versailles; 63-74. https://agritrop.cirad.fr/575562/

MAEP. 2013. Document d'orientation stratégique de la filière coton au Togo. MAEP, Togo, 60 p.

Mahamat H, Belko N, Cisse N, Sine B, Ndoye I. 2014. Amélioration de l'adaptation à la sécheresse chez le niébé (Vigna unguiculata L. Walpers). Journal of Applied Biosciences, 77: 6550-6563. DOI: 10.4314/jab.v77i1.12

Ouédraogo M, Dembélé Y, Somé L. 2010. Perceptions et stratégies d'adaptation aux changements des précipitations : cas des paysans du Burkina Faso. Sécheresse, 21(2): 87-96. DOI: 10.1684/sec.2010.0244

Pettigrew WT. 2004. Moisture deficit effects on cotton lint yield, yield components, and boll distribution. Agronomy Journal,
96: 377-383. DOI: https://doi.org/10.2134/agronj2004.3770

Rahman M, Ullah I, Ahsraf M, Stewart JM, Zafar Y. 2008. Genotypic variation for drought tolerance in cotton. Agron. Sustain. Dev., 28: 439-447. DOI: 10.1051/Agro: 2007041

Sezener V, Basal H, Peynircioglu C, Gurbuz T, Kizilkaya K. 2015. Screening of cotton cultivars for drought tolerance under field conditions. Turk J. Field Crops, 20(2): 223-232. DOI: $10.17557 /$ tjfc.57032

Sissoko F, Sidiki Diarra S, Traore M. 2020. Le semis direct sous couverture végétale : une opportunité de mise en place rapide du cotonnier en culture pluviale au Mali. Int. J. Biol. Chem. Sci., 14(3): 722-738. DOI: $10.4314 /$ ijbcs.v14i3.7

Sissoko F, Koulibaly B, Dagbenonbakin GD, Naïtormbaïde M, Fayalo RDG, Amonmide I, Traore KL, Dias Coelho ACA, Gilvan B, Ferreira B, Bogiani JC, Di Stéfano JG, Lamas FM, Carvalho MCS. 2013. Système de semis direct sous couverture végétale. Embrapa, Brésil.

Some PP, Hien E, Tozo K, Zombre G, Dianou D. 2010. Effets de six composts sur les réponses physiologiques, biochimiques et agronomiques du niébé Vigna unguiculata L. Walp var. KVX. 61.1. au déficit hydrique. Int. J. Biol. Chem. Sci., 8(1): $\quad 31-45$. DOI: http://dx.doi.org/10.4314/ijbcs.v8i1.4

Sultan B, Janicot S, Christian Baron C, Dingkuhn D, Muller B, Traoré S, Sarr B. 2008. Les impacts agronomiques du climat en Afrique de l'Ouest : une illustration des problèmes majeurs. Sécheresse, 19(1): 29-37.

Zare M, Mohammadifard GR, Bazrafshan F, Zadehbagheri M. 2014. Evaluation of cotton (Gossypium hirsutum L.) genotypes to drought stress. Int. Jour. of Biosci., 4(12): 158-166. 